\title{
Optically Generated Ultrasound for Intracoronary Imaging
}

\author{
Callum D. Little ${ }^{1,2 * \dagger}$, Richard J. Colchester ${ }^{2,3 \dagger}$, Sacha Noimark ${ }^{2,3}$, Gavin Manmathan ${ }^{1}$, \\ Malcolm C. Finlay ${ }^{2,3,4}$, Adrien E. Desjardins ${ }^{2,3}$ and Roby D. Rakhit ${ }^{1,2}$ \\ ${ }^{1}$ Department of Cardiovascular Medicine, Royal Free NHS Foundation Trust, London, United Kingdom, \\ ${ }^{2}$ Wellcome-Engineering \& Physical Sciences Research Council (EPSRC) Centre for Interventional and Surgical Sciences, \\ London, United Kingdom, ${ }^{3}$ Department of Medical Physics and Bioengineering, University College London, London, \\ United Kingdom, ${ }^{4}$ William Harvey Cardiovascular Research Institute, Queen Mary University of London and Barts Health \\ Centre London, London, United Kingdom
}

Conventional intravascular ultrasound (IVUS) devices use piezoelectric transducers to electrically generate and receive US. With this paradigm, there are numerous challenges that restrict improvements in image quality. First, with miniaturization of the transducers to reduce device size, it can be challenging to achieve the sensitivities and bandwidths

OPEN ACCESS

Edited by:

Christos Bourantas,

Barts Health NHS Trust,

United Kingdom

Reviewed by:

Erhan Tenekecioglu,

University of Health Sciences, Turkey Julien Bec,

University of California, Davis, United States

*Correspondence: Callum D. Little c.little@ucl.ac.uk

${ }^{\dagger}$ These authors have contributed equally to this work and share first authorship

Specialty section:

This article was submitted to Cardiovascular Imaging, a section of the journal Frontiers in Cardiovascular Medicine

Received: 09 January 2020 Accepted: 04 September 2020 Published: 14 October 2020

Citation:

Little CD, Colchester RJ, Noimark S, Manmathan G, Finlay MC, Desjardins AE and Rakhit RD (2020) Optically Generated Ultrasound for Intracoronary Imaging. Front. Cardiovasc. Med. 7:525530. doi: 10.3389/fcvm.2020.525530 required for large tissue penetration depths and high spatial resolution. Second, complexities associated with manufacturing miniaturized electronic transducers can have significant cost implications. Third, with increasing interest in molecular characterization of tissue in-vivo, it has been challenging to incorporate optical elements for multimodality imaging with photoacoustics (PA) or near-infrared spectroscopy (NIRS) whilst maintaining the lateral dimensions suitable for intracoronary imaging. Optical Ultrasound (OpUS) is a new paradigm for intracoronary imaging. US is generated at the surface of a fiber optic transducer via the photoacoustic effect. Pulsed or modulated light is absorbed in an engineered coating on the fiber surface and converted to thermal energy. The subsequent temperature rise leads to a pressure rise within the coating, which results in a propagating ultrasound wave. US reflections from imaged structures are received with optical interferometry. With OpUS, high bandwidths $(31.5 \mathrm{MHz})$ and pressures $(21.5$ $\mathrm{MPa}$ ) have enabled imaging with axial resolutions better than $50 \mu \mathrm{m}$ and at depths $>20 \mathrm{~mm}$. These values challenge those of conventional $40 \mathrm{MHz}$ IVUS technology and show great potential for future clinical application. Recently developed nanocomposite coating materials, that are highly transmissive at light wavelengths used for PA and NIRS light, can facilitate multimodality imaging, thereby enabling molecular characterization.

Keywords: optical ultrasound, OPUS, optoacoustics, imaging, intravascular ultrasound, IVUS

\section{INTRODUCTION}

Intravascular imaging has the ability to provide invaluable anatomical information to facilitate the treatment of coronary artery disease (1). Additionally, molecular compositional analysis of atheroscletoric plaque disease may help to identify targets for intervention (2). The two leading technologies in this field for microstructural imaging are Optical Coherence Tomography (OCT) and Intravascular Ultrasound (IVUS). OCT images are obtained using broadband near-infrared light (wavelengths typically in the vicinity of $1,310 \mathrm{~nm}$ ), with reflections from tissue detected interferometrically. High axial $(10 \mu \mathrm{m})$ and lateral $(20 \mu \mathrm{m})$ resolutions allow for assessments of 
both vascular endothelium and plaque structural components, albeit with limited tissue penetration (typically $<2 \mathrm{~mm}$ ) (3). IVUS is a modality analogous to OCT, in which ultrasonic reflections from tissue are detected. Typical commercial IVUS imaging catheters operate with transducer frequencies centered at around $40 \mathrm{MHz}$, providing an axial resolution of $100-150 \mu \mathrm{m}$, a lateral resolution of $200 \mu \mathrm{m}$ and a tissue penetration of 4$8 \mathrm{~mm}$ (3). IVUS has established roles in sizing vessels, detecting calcium, and guiding optimal stent expansion. IVUS has seen exciting technological advancements in recent years, including the use of dual frequency probes to allow for high resolution imaging (4) and combinations with near-infrared spectroscopy (NIRS) (5) or photoacoustics (PA) (6) to provide hybrid imaging with molecular compositional analysis.

With contemporary IVUS devices, ultrasound (US) is generated and received electrically, using piezoelectric transducers. Whilst this electronic platform is well-established within the field of interventional cardiology, there are limitations that preclude its broader clinical use. First, with very small elements (e.g., diameter $<150 \mu \mathrm{m}$ ), it can be challenging to achieve adequate sensitivity and bandwidth for high penetration depth and high resolution tissue imaging. Second, the complexities associated with fabricating and electrically connectorising broadband piezocomposite transducers can result in high manufacturing costs. Third, the broader applicability of electronic interventional devices in the coronary catheterization suite is challenged by sensitivities to electromagnetic interference and lack of MRI compatibility (7).

Optical ultrasound (OpUS) imaging probes, in which transmission and reception are both performed with light, are emerging as alternatives to their electrical counterparts. They offer several key advantages, including the potential to generate and detect the broadband US fields (tens of $\mathrm{MHz}$ ) required for high resolution intravascular imaging (8) and immunity to EM interference. Moreover, optical fibers used for ultrasound transmission and reception can provide the required level of miniaturization for minimally invasive use and have costs that lend themselves to disposable devices. Furthermore, the use of optical fibers allows for the integration of complementary imaging and therapeutic modalities without compromising the device size or performance (9). In this review, we describe the application of OpUS to coronary imaging, including preclinical data acquired using this technology, and future translational applications.

\section{Optical Ultrasound Generation and Reception}

With optical ultrasound (OpUS) the generation of US occurs via the photoacoustic effect at the surface of a fiber optic transducer (8), wherein pulsed or modulated excitation light is absorbed in a coating and converted to thermal energy. The transient heat rise leads to a corresponding pressure rise which propagates as an ultrasound wave. The bandwidth of this wave depends on the temporal characteristics of the excitation light. In general, the bandwidth can be increased by decreasing the duration of excitation light pulses; however, in practice, these increases are limited by frequency-dependent ultrasound attenuation within the coating (10) and within blood and vascular tissue (11). To achieve efficient optical-US transduction, a material with a high optical absorption coefficient and a high thermal expansion coefficient is desirable. Studies to date have highlighted the efficacy of composite materials that comprise optical absorbers integrated within elastomers. With these considerations in mind, several nanocomposite materials have been explored, including carbonaceous materials (8), metallic nanoparticles (9), and organic pigments (9). Carbonaceous materials including carbon black (12), carbon nanofibers (13), candle soot (14), carbon nanotubes (15), and graphene (16) have high optical absorption across the visible and near-infrared wavelength ranges. Metallic nanoparticles such as gold, exhibit a relatively narrow optical absorbing window. Organic pigments such as crystal violet can display poor photostability with repeated usage causing photobleaching with loss of acoustic conversion efficiency (9). Noimark et al. showed for the first time that functionalized multiwalled carbon nanotube-polydimethylsiloxane (PDMS) composites can give rise to pressures of $21.5 \mathrm{MPa}$ at the coating surface (the highest recorded pressure from a fiber optic transmitter, to our knowledge) and bandwidths of $39.8 \mathrm{MHz}$ (15). These high pressures enable high imaging penetration depths, and the broad bandwidths give rise to high axial resolution. A small coating thickness can be important to minimize acoustic attenuation within nanocomposite materials (10). To this end, several methods have been explored for depositing nanocomposite materials onto the distal surface of the fiber optic transducer, including spin-coating (17), electrospinning (18), and dip-coating (15). With the latter method, a "bottom up" approach typically involves coating the substrate with an optical absorber and a subsequent polymer overcoat (for instance, PDMS). Control of the thickness of the nanocomposite region comprising the optical absorber and the polymer, and the total thickness of the polymer, is determined by both the viscosity of the material, which can be altered through the use of solvents, and the dipping speed of the optical fiber.

To allow for real-time pulse-echo US imaging, fiber-optic transmitters have been be paired with high-finesse FabryPérot fiber-optic receivers [(19); Figure 1A]. These receivers, fabricated on the distal ends of optical fibers, comprise an epoxy dome with high-reflectivity coatings on both the planar and domed surfaces. When an ultrasound wave impinges on the dome, it causes nanometer-scale deformations in the dome surface. These deformations are measured using laser interferometry. Such devices are capable of measuring pressures lower than 100 Pascals, making them highly sensitive to ultrasound reflections and ideally suited to minimally invasive imaging. In contrast to piezoelectric receivers, whose sensitivities fall off with decreasing element size, fiber optic receivers can maintain their sensitivity even at scales of tens or hundreds of micrometers. Their small lateral dimensions $(<250 \mu \mathrm{m}$ outer diameter) enable integration into intracoronary imaging devices, and their large bandwidths yield high imaging performance [e.g., axial resolution better than $60 \mu \mathrm{m}$ and $\mathrm{cm}$-scale imaging depths without temporal averaging (20)]. Several other fiber optic reception technologies are promising for intracoronary imaging, 

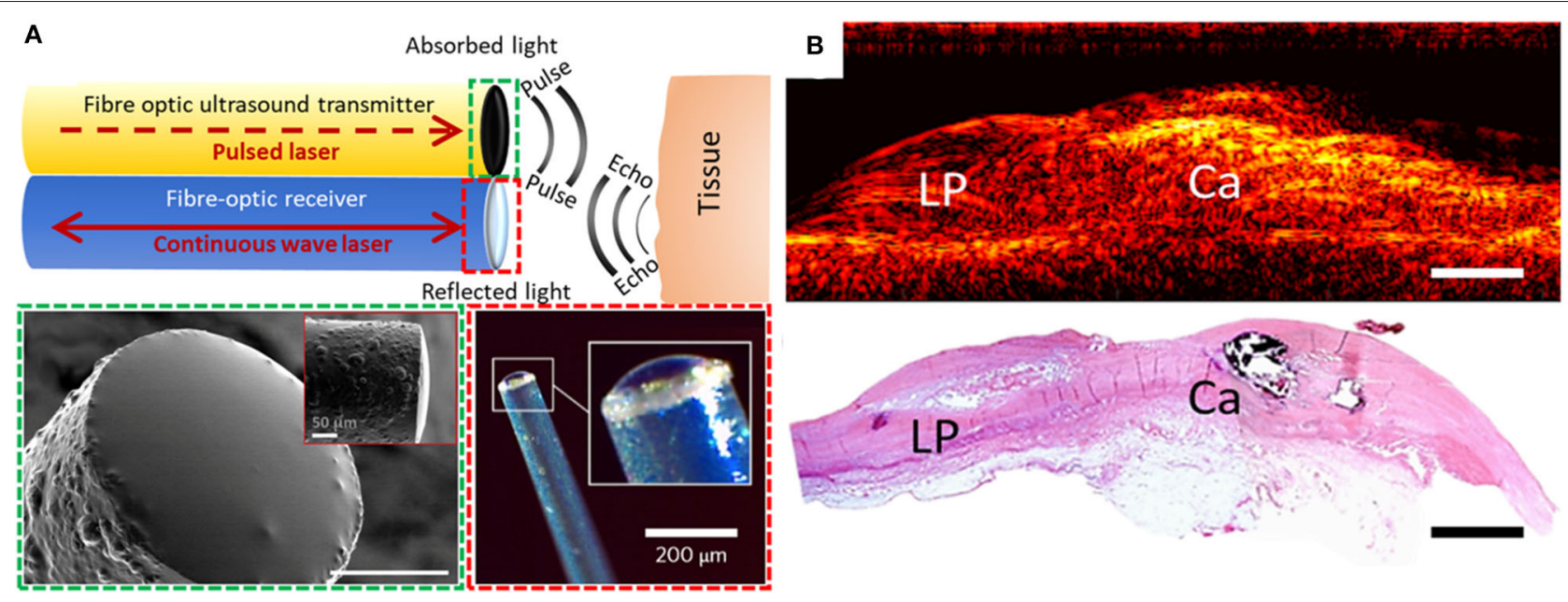

FIGURE 1 | (A) Generation and reception of OpUS. The schematic (top) includes both a transmitter and a receiver. Lower-left inset (dashed green box): scanning electron microscopy image of the transmitter fiber-tip coated with nanocomposite (scale bar: $50 \mu \mathrm{m})$ (15). Lower-right inset (dashed red box): optical microscopy of the fiber-optic receiver (19). (B) 2D longitudinal M-mode OpUS imaging of ex-vivo human coronary artery tissue with a lipid pool (LP), calcification (Ca), and corresponding histology (haematoxylin and eosin staining) (scale bars: $2 \mathrm{~mm}$ ).

including fiber Bragg gratings (21-23) and microring resonators $(24,25)$.

With OpUS, high bandwidths and pressures have enabled imaging with resolutions better than $50 \mu \mathrm{m}$ and tissue penetration depths $>20 \mathrm{~mm}$ (20). These values challenge those of conventional $40 \mathrm{MHz}$ IVUS technology and show great potential for future clinical application. As initial demonstrations of the viability of OpUS for in-vivo clinical imaging, forward-viewing configurations have been used. With these configurations, ultrasound was transmitted ahead of the optical fiber, in a direction colinear with the optical fiber axis. Axial and lateral resolutions of $<60$ and $<90 \mu \mathrm{m}$, respectively, were achieved (26). A forward-viewing probe that was integrated within a transseptal puncture needle was used to obtain the first in vivo intracardiac images with OpUS (27). Recently, the first OpUS images of ex-vivo human coronary tissue samples (Figure 1B) were acquired and compared to histology. Numerous features of atherosclerotic plaque were identifiable, including a lipid pool, a calcified nodule, and the different layers of the vessel wall. Several other configurations relevant to intracoronary imaging have subsequently been developed, including side-viewing rotational imaging (20) and hybrid multimodality imaging (9).

\section{OpUS Structural Imaging}

Commercial intravascular imaging systems can employ rotational pullbacks of side-viewing probes in order to achieve cross-sectional imaging (with phased-array probes as alternatives). An analogous rotational implementation with OpUS can be realized with ultrasound transmitted perpendicular to the device axis. In a recent probe, perpendicular ultrasound transmission was made possible with an optically-absorbing nanocomposite coating extending perpendicular to the axis of the optical fiber that transmitted excitation light, in conjunction with a $45^{\circ}$ mirror (Figure 2A). This optical fiber was connected to a rotary junction in order to allow for circumferential imaging, whilst the omnidirectional fiber-optic receiver remained stationary. The configuration of this imaging device had dimensions suitable for intracoronary imaging, with a maximum lateral dimension $<1.25 \mathrm{~mm}$ at the distal tip. Image acquisition occurred with a frame rate of 5 frames per second. The imaging fidelity of the device was investigated with ex-vivo swine carotid tissue [(20); Figure 2B]. The broad bandwidths achieved ( $-6 \mathrm{~dB}$ bandwidth of $31.3 \mathrm{MHz}$ ), in conjunction with depth-dependent digital frequency filtering, allowed for both high axial resolutions at shallow depths and deep tissue visualization. Axial and lateral resolutions at a distance of $3 \mathrm{~mm}$ from the probe were superior to $50 \mu \mathrm{m}$ and $15^{\circ}$. This device sets the stage for future devices with rotational lengths and encapsulation suitable for in vivo intracoronary imaging.

\section{Multi-Modality US and Photoacoustic Imaging}

Recently there has been intense interest in multi-modality intravascular imaging probes that provide complementary structural and molecular information. These probes include combinations such as US with photoacoustic (PA) imaging $(6,28)$ or with near-infrared spectroscopy (NIRS) $(5,29)$. A potential strong advantage of OpUS probes, as compared to their electronic counterparts, is that the optical fibers used to generate and receive ultrasound can also be used to transmit light for PA and NIRS. These efficiencies, which can be achieved through the use of nanocomposite fiber tip coatings that are absorbing at certain wavelengths and transmissive at others, could be valuable both for achieving high levels of miniaturization and cost reductions. In a study by Noimark and Colchester et al., gold nanoparticle composites and organic dye composites, applied to the distal ends 


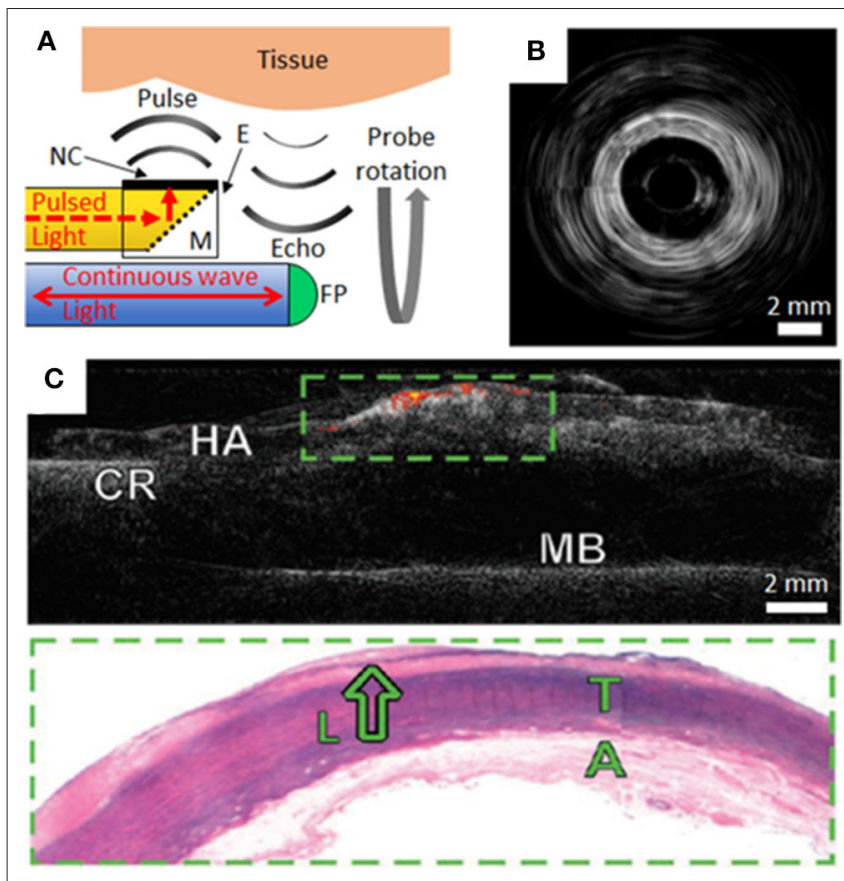

FIGURE 2 | (A) Schematic of side-viewing optical ultrasound imaging probe with pulsed excitation light transmitted along an optical fiber (yellow), reflected at a mirror (M) and absorbed at the nanocomposite (NC) coating surface of an epoxy-based (E) optical transducer housing. Ultrasound pulses reflect from tissue and the echoes are received through interferometric interrogation of the Fabry-Pérot (FP) cavity at the distal end of the receiving optical fiber (blue). The probe is rotated about its axis to achieve circumferential imaging. (B) Rotational optical ultrasound images obtained within an ex-vivo swine carotid artery (20). (C) Co-registered optical ultrasound (gray-scale) and photoacoustic images of human aortic tissue ( $\mathrm{HA})$ using a gold nanoparticle/PMDS composite transmitter. Areas of lipid are displayed as a color signal. The tissue was secured to a cork ring (CR) and metal base (MB) for imaging. Corresponding histology of the green boxed area highlights a lipid pool (L), tunica media (T) and adventitia (A) (9).

of optical fibers within an imaging probe, were used to generate ultrasound for OpUS (532 nm wavelength) and to transmit light for PA imaging $(1,210 \mathrm{~nm})$ from a single optical fiber (9). Coregistered ultrasound and photoacoustic images of an ex-vivo diseased human aorta were acquired. PA imaging provided molecular contrast for lipid rich coronary plaque, which was overlaid on the acquired ultrasound images (Figure 2C). Whilst the use of multi-modality OpUS for intracoronary imaging is at an early stage, there are strong indications that it could allow for measuring the plaque burden, which in turn could be valuable for guiding stent placement and for improving our understanding of the pathophysiology of coronary artery disease.

\section{DISCUSSION}

Direct comparison of OpUS to established intracoronary imaging devices is challenging at present due to a lack of in-vivo clinical data. Whilst detection of lipid and calcium by OpUS has been demonstrated (Figure 1B), further work is required to assess the efficacy of this technology for accurately sizing vessel diameter, luminal area, optimizing stent expansion, and detection of high risk atherosclerotic plaque morphological features including fibrous cap thickness, neovascularisation and macrophage infiltration. Nevertheless, OpUS potentially offers several prominent benefits over current generation electronically generated ultrasound. The axial resolution could be enhanced by the use of modulated excitation light that emphasizes higher ultrasound frequencies. Improvements to the lateral resolution could be achieved with curved transducers to achieve a focussed beam and with shorter excitation light pulses to increase the central frequency, and potentially with deep-learning beamforming for sub-sampled data (30). In order to improve image quality further refinements in image post-processing are possible, including complex filtering and reconstruction approaches, as well as adaptive frequency filtering to allow discrimination between acoustically dissimilar tissues (31-35). Significant increases in image acquisition rate are readily achievable. The rotational OpUS setup demonstrated to date (20) was limited to 5 frames per second by the data transfer rate and computation times, but further engineering developments with existing excitation lasers could potentially increase imaging rates to over 100 frames per second without compromising lateral resolution. To enable more rapid circumferential imaging for clinical translation, mechanical elements such as torque coils could be incorporated into the imaging probes, as used with OCT catheters. It is envisaged that lubricating fluid will be necessary to reduce frictional forces generated by the rotational components and to facilitate ultrasound coupling. Looking beyond the paradigm of rotating a single optical transducer, a phased array analogous to solid-state IVUS systems could be envisaged; however, it is likely to be challenging to achieve a high density of optical transducer elements within a coronary imaging device without compromising the device profile or deliverability. It is conceivable that spatial light modulators for controlling the propagation of laser light through multimode optical fibers (36) could be used in this context. A forward-looking volumetric probe analogous to electronic IVUS versions (37) could be envisaged; however, the spatial resolution would be highly dependent on the geometry and spatial configuration of the transducer elements.

Looking beyond aforementioned probes for vascular imaging that have been demonstrated at a pre-clinical level, there are uncharted frontiers in which OpUS probes could be extended to include new capabilities. They include enhanced diagnosis via pairing with OCT, photoacoustic imaging (38), and nearinfrared fluorescence (NIRF) (39); additionally, they include therapeutic monitoring during atherectomy (40) and device tracking with the inclusion of fiber optic shape sensing (41). Speculatively, the inclusion of OCT could be enabled with a double-clad optical fiber, with ultrasound reception and OCT performed with light transmitted along the single-mode inner core and ultrasound transmission with the multi-mode outer core. Optically-generated ultrasound represents an exciting 
development in the field of intravascular imaging. Whilst many steps along the translational path remain, there is strong potential to unite many modalities into a single fiber optic probe that could have broad applicability in cardiovascular medicine and beyond.

\section{AUTHOR CONTRIBUTIONS}

$\mathrm{CL}, \mathrm{RC}$, and $\mathrm{AD}$ co-wrote the paper. $\mathrm{SN}$ provided advice on descriptions of the nanocomposite materials. GM, MF, and $\mathrm{RR}$ provided advice on the clinical applications of OpUS.

\section{REFERENCES}

1. Neumann FJ, Sousa-Uva M, Ahlsson A, Alfonso F, Banning AP, Benedetto U, et al. 2018 ESC/EACTS guidelines on myocardial revascularization. Eur Heart J. (2019) 40, 87-165. doi: 10.1093/ejurheartj/ehy394

2. Stone GW, Maehara A, Lansky AJ, de Bruyne B, Cristea E, Mintz GS, et al. a prospective natural-history study of coronary atherosclerosis. $\mathrm{N} \mathrm{Engl} \mathrm{J} \mathrm{Med.}$ (2011) 364:226-35. doi: 10.1056/nejmoa1002358

3. Koganti S, Kotecha T, Rakhit RD. Choice of intracoronary imaging: when to use intravascular ultrasound or optical coherence tomography. Interv Cardiol Rev. (2016) 11:6-11. doi: 10.15420/icr.2016:6:1

4. Chin CY, Maehara A, Fall K, Mintz GS, Ali ZA. Imaging comparisons of coregistered native and stented coronary segments by high-definition $60-\mathrm{MHz}$ intravascular ultrasound and optical coherence tomography. JACC Cardiovasc Interv. (2016) 9:1305-6. doi: 10.1016/j.jcin.2016.04.011

5. Waksman R, Di Mario C, Torguson R, Ali ZA, Singh V, Skinner $\mathrm{WH}$, et al. Identification of patients and plaques vulnerable to future coronary events with near-infrared spectroscopy intravascular ultrasound imaging: a prospective, cohort study. Lancet. (2019) 294:1629-37. doi: 10.1016/S0140-6736(19)31794-5

6. Cao Y, Kole A, Hui J, Zhang Y, Mai J, Alloosh M, et al. Fast assessment of lipid content in arteries in vivo by intravascular photoacoustic tomography. Sci Rep. (2018) 8:2400. doi: 10.1038/s41598-018-20881-5

7. Raman VK, Lederman RJ. Interventional cardiovascular magnetic resonance imaging. Trends Cardiovasc Med. (2007) 17:196-202. doi: $10.1016 /$ j.tcm.2007.05.003

8. Colchester RJ, Mosse CA, Bhachu DS, Bear JC, Carmalt CJ, Parkin IP, et al. Laser-generated ultrasound with optical fibres using functionalised carbon nanotube composite coatings. Appl Phys Lett. (2014) 104:173502. doi: $10.1063 / 1.4873678$

9. Noimark S, Colchester RJ, Poduval RK, Maneas E, Alles EJ, Zhao $\mathrm{T}$, et al. Polydimethylsiloxane composites for optical ultrasound generation and multimodality imaging. Adv Funct Mater. (2018) 28:1-16. doi: 10.1002/adfm.201704919

10. Alles EJ, Heo J, Noimark S, Colchester RJ, Parkin IP, Baac HW, et al. Acoustical characterisation of carbon nanotube-loaded polydimethylsiloxane used for optical ultrasound generation. In: IEEE International Ultrasonics Symposium, IUS. Washington, DC, (2017). p. 1-4. doi: 10.1109/ULTSYM.2017.8092343

11. Treeby BE, Zhang EZ, Thomas AS, Cox BT. Measurement of the ultrasound attenuation and dispersion in whole human blood and its components from 0-70 MHz. Ultrasound Med Biol. (2011) 37:289-300. doi: 10.1016/j.ultrasmedbio.2010.10.020

12. O’Donnell M, Hou Y, Kim JS, Ashkenazi S, Huang SW, Guo LJ. Optoacoustic generation of high frequency sound for 3-D ultrasonic imaging in medicine. Eur Phys J Spec Top. (2008) 153:53-8. doi: 10.1140/epjst/e2008-00392-9

13. Hsieh BY, Kim J, Zhu J, Li S, Zhang X, Jiang X. A laser ultrasound transducer using carbon nanofibers-polydimethylsiloxane composite thin film. Appl Phys Lett. (2015) 106:021902. doi: 10.1063/1.4905659

14. Aytac-Kipergil E, Alles EJ, Pauw HC, Karia J, Noimark S, Desjardins AE. Versatile and scalable fabrication method for laser-generated focused ultrasound transducers. Opt Lett. (2019) 44:6005-8. doi: 10.1364/ol.44. 006005
All authors contributed to the article and approved the submitted version.

\section{FUNDING}

$\mathrm{CL}, \mathrm{RC}, \mathrm{AD}$, and RR gratefully acknowledge funding by the National Institute for Health Research UCL Biomedical Research Centre and the Wellcome/EPSRC Centre for Interventional and Surgical Sciences (WEISS) (203145Z/16/Z; NS/A000050/1). RC was funded by the Royal Academy of Engineering under the Research Fellowship scheme.

15. Noimark S, Colchester RJ, Blackburn BJ, Zhang EZ, Alles EJ, Ourselin $\mathrm{S}$, et al. Carbon-nanotube-PDMS composite coatings on optical fibers for all-optical ultrasound imaging. Adv Funct Mater. (2016) 26:8390-6. doi: 10.1002/adfm.201601337

16. Colchester RJ, Alles EJ, Desjardins AE. A directional fibre optic ultrasound transmitter based on a reduced graphene oxide and polydimethylsiloxane composite. Appl Phys Lett. (2019) 114:113505. doi: 10.1063/1.50 89750

17. Biagi E, Cerbai S, Masotti L, Belsito L, Roncaglia A, Masetti G, et al. MOMS technology for fully fiber optic ultrasonic probes: a proposal for virtual biopsy. In: Proceedings of IEEE Sensors. Waikoloa, HI (2010). p 1156-60. doi: 10.1109/ICSENS.2010.5690824

18. Poduval RK, Noimark S, Colchester RJ, Macdonald TJ, Parkin IP, Desjardins AE, et al. Optical fiber ultrasound transmitter with electrospun carbon nanotube-polymer composite. Appl Phys Lett. (2017) 110:223701. doi: 10.1063/1.4984838

19. Guggenheim JA, Li J, Allen TJ, Colchester RJ, Noimark S, Ogunlade O, et al. Ultrasensitive plano-concave optical microresonators for ultrasound sensing. Nat Photonics. (2017) 11:714-9. doi: 10.1038/s41566-017-0027-x

20. Colchester RJ, Little C, Dwyer G, Noimark S, Alles EJ, Zhang EZ, et al. All-optical rotational ultrasound imaging. Sci Rep. (2019) 9:5576. doi: 10.1038/s41598-019-41970-z

21. Rosenthal A, Razansky D, Ntziachristos V. High-sensitivity compact ultrasonic detector based on a pi-phase-shifted fiber Bragg grating. Opt Lett. (2011) 36:1833-5. doi: 10.1364/ol.36.001833

22. Wu Q, Okabe Y. High-sensitivity ultrasonic phase-shifted fiber Bragg grating balanced sensing system. Opt Express. (2012) 20:28353-62. doi: 10.1364/oe.20.028353

23. Tian J, Zhang Q, Han M. Distributed fiber-optic laser-ultrasound generation based on ghost-mode of tilted fiber Bragg gratings. Opt Express. (2013) 21:6109-14. doi: 10.1364/oe.21.006109

24. Dong B, Chen S, Zhang Z, Sun C, Zhang HF. Photoacoustic probe using a microring resonator ultrasonic sensor for endoscopic applications. Opt Lett. (2014) 39:4372-5. doi: 10.1364/ol.39.004372

25. Hsieh B-Y, Chen S-L, Ling T, Guo LJ, Li P-C. Integrated intravascular ultrasound and photoacoustic imaging scan head. Opt Lett. (2010) 35:28924. doi: 10.1364/ol.35.002892

26. Colchester RJ, Zhang EZ, Mosse CA, Beard PC, Papakonstantinou I, Desjardins AE. Broadband miniature optical ultrasound probe for high resolution vascular tissue imaging. Biomed Opt Express. (2015) 6:1502. doi: 10.1364/boe.6.001502

27. Finlay MC, Mosse CA, Colchester RJ, Noimark S, Zhang EZ, Ourselin S, et al. Through-needle all-optical ultrasound imaging in vivo: a preclinical swine study. Light Sci Appl. (2017) 6:e17103-7. doi: 10.1038/lsa.2017.103

28. Jansen K, Van Soest G, van der Steen AFW. Intravascular photoacoustic imaging: a new tool for vulnerable plaque identification. Ultrasound Med Biol. (2014) 40:1037-48. doi: 10.1016/j.ultrasmedbio.2014. 01.008

29. Roleder T, Kovacic JC, Ali Z, Sharma R, Cristea E, Moreno P, et al. Combined NIRS and IVUS imaging detects vulnerable plaque using a single catheter system: a head-to-head comparison with OCT. EuroIntervention. (2014) 10:303-11. doi: 10.4244/EIJV10I3A53 
30. Simson W, Paschali M, Navab N, Zahnd G. Deep learning beamforming for sub-sampled ultrasound data. In: IEEE International Ultrasonics Symposium, IUS. Kobe (2018). p. 1-4. doi: 10.1109/ULTSYM.2018.8579818

31. Park J, Kang JB, Chang JH, Yoo Y. Speckle reduction techniques in medical ultrasound imaging. Biomed Eng Lett. (2014) 432-40. doi: 10.1007/s13534-014-0122-6

32. Lediju Bell MA, Goswami R, Kisslo JA, Dahl JJ, Trahey GE. Short-Lag Spatial Coherence Imaging of Cardiac Ultrasound Data: Initial Clinical Results. Ultrasound Med Biol. (2013) 39:1861-74. doi: 10.1016/j.ultrasmedbio.2013.03.029

33. Rouyer J, Varray F, Pozo E, Basset O, Cachard C, Lavarello R. Evaluation of a frequency-domain ultrasonic imaging attenuation compensation technique. In: Proceedings of the Annual International Conference of the IEEE Engineering in Medicine and Biology Society, EMBS. Milan (2015). p. 15603. doi: 10.1109/EMBC.2015.7318670

34. Varray F, Cachard C, Kybic J, Novell A, Bouakaz A, Basset O. A multifrequency approach to increase the native resolution of ultrasound images. In: European Signal Processing Conference. Bucharest (2012) p. 2733-7.

35. Allman D, Reiter A, Bell MAL. Photoacoustic source detection and reflection artifact removal enabled by deep learning. IEEE Trans Med Imaging. (2018) 37:1464-77. doi: 10.1109/TMI.2018.2829662

36. Cižmár T, Dholakia K. Shaping the light transmission through a multimode optical fibre: complex transformation analysis and applications in biophotonics. Opt Express. (2011) 19:18871-84. doi: 10.1364/oe.19.018871

37. Guldiken R, Zahorian J, Gurun G, Qureshi MS, Balantekin M, Tekes C, et al. Forward-looking IVUS imaging using a dual-annular ring CMUT array: Experimental results. In: Proceedings - IEEE Ultrasonics Symposium. New York, NY (2007). p. 1247-50. doi: 10.1109/ULTSYM.2007.313
38. van Soest G, Marcu L, Bouma BE, Regar E. Intravascular imaging for characterization of coronary atherosclerosis. Curr Opin Biomed Eng. (2017) 3:1-12. doi: 10.1016/j.cobme.2017.07.001

39. Yoo H, Kim JW, Shishkov M, Namati E, Morse T, Shubochkin R, et al. Intraarterial catheter for simultaneous microstructural and molecular imaging in vivo. Nat Med. (2011) 171680-4. doi: 10.1038/nm.2555

40. Rawlins J, Din JN, Talwar S, O'Kane P. Coronary intervention with the excimer laser: review of the technology and outcome data. Interv Cardiol Rev. (2016) 11:27-32. doi: 10.15420/icr.2016:2:2

41. Shi C, Giannarou S, Lee SL, Yang GZ. Simultaneous catheter and environment modeling for Trans-catheter Aortic Valve Implantation. In: 2014 IEEE International Conference on Intelligent Robots and Systems. (2014) p. 20249. doi: 10.1109/IROS.2014.6942832

Conflict of Interest: AD, RC, and MF are shareholders of Echopoint Medical Ltd.

The remaining authors declare that the research was conducted in the absence of any commercial or financial relationships that could be construed as a potential conflict of interest.

Copyright (c) 2020 Little, Colchester, Noimark, Manmathan, Finlay, Desjardins and Rakhit. This is an open-access article distributed under the terms of the Creative Commons Attribution License (CC BY). The use, distribution or reproduction in other forums is permitted, provided the original author(s) and the copyright owner(s) are credited and that the original publication in this journal is cited, in accordance with accepted academic practice. No use, distribution or reproduction is permitted which does not comply with these terms. 